\title{
A method to determine winding losses in integrated inductors and separate skin and proximity effects
}

\author{
A. Abderahim ${ }^{1}$ - M. Koularambaye ${ }^{1} \cdot$ J. P. Chatelon ${ }^{1} \cdot$ S. Capraro $^{1}$ (i) $\cdot$ D. Piétroy ${ }^{1}$ ·. J. Rousseau ${ }^{1}$
}

Received: 21 February 2020 / Accepted: 6 May 2020 / Published online: 13 May 2020

(c) Springer Nature Switzerland AG 2020

\begin{abstract}
This paper presents a method to determine winding losses from scattering matrix parameters in planar inductors. Winding losses are taken into account by a dependent frequency resistance. This method allows losses to be separated in two parts for coreless inductors: skin effect and proximity effect. In high frequencies both software, like High Frequency Structure Simulator, and equipment, like Vector Network Analyzer, provide scattering parameters that allow both impedance or admittance matrices to be calculated. Then, parameters of an electric equivalent circuit can be calculated. The resistance value taking into account both skin and proximity effects is determined in three frequency domains. The first domain is at very low frequencies or at direct current. The second domain is at low-medium frequencies where the capacitive couplings are negligible. The last domain is at the resonant frequencies of the admittance matrix parameters, so at high frequencies. The presented approach is different from those encountered in literature and allows the series resistance from measured or simulated scattering matrix parameters to be determined.
\end{abstract}

Keywords Integrated planar devices · Magnetic component · Scattering parameters · Skin and proximity effects . Winding losses

\section{Introduction}

Power supplies for embedded equipment require high efficiencies, both low weights and sizes. For some years, there has been a strong demand for miniaturization and integration of magnetic components like inductors and transformers for DC-DC power converters. In order to reach high efficiencies, both losses in active and passive components have to be reduced. This article deals with the determination of copper losses versus frequency in integrated magnetic components. Copper losses are modeled by using a serial resistance $r_{(f)}$ that depends on the frequency. The resistance $r_{(f)}$ takes into account both skin and proximity effects. In addition, our approach makes it possible to separate skin and proximity effects. In the past, numerous works were devoted to determination, calculation of copper losses for wound windings using round wires, Litz wires or foils [1-4]. Some authors investigated winding losses in low-profile magnetic components [5].

Papers related to determination of winding losses in integrated inductors are not so many [6-12]. The analytical expression (1) proposed by Yue [6] which takes into account skin effects is used by most authors.

$R_{e p}=L \frac{\rho}{w \delta\left(1-e^{-t / \delta}\right)}$

$\mathbf{R}_{\text {ep }}$ series resistance, $\mathbf{L}$ length of the wire, $\boldsymbol{\rho}$ copper resistivity, w conductor width, $\boldsymbol{\delta}$ skin depth, $\mathbf{t}$ conductor thickness.

On the other hand, Kuhn [7] studied ac winding losses in planar spiral inductors on silicon. They expressed the AC

S. Capraro, stephane.capraro@univ-st-etienne.fr | ${ }^{1}$ Université de Lyon, CNRS UMR 5516, Laboratoire Hubert Curien, Université JeanMonnet, 42000 Saint-Étienne, France. 
resistance as a function of the DC resistance and a critical pulsation $\omega_{c r i}$ :

$R_{A C}=R_{D C}\left[1+\frac{1}{10}\left(\frac{\omega}{\omega_{\text {crit }}}\right)^{2}\right]$

$\omega_{\text {crit }}=\frac{3,1(w+s)}{\mu_{0} w^{2}} R_{\text {square }} R_{\text {square }}=\frac{\rho}{t}$ and $\omega=2 \pi f$

s space between two consecutive turns, $\mathbf{f}$ frequency.

In order to take into account both skin and proximity effects, the pulsation $\omega_{\text {crit }}$ depends on $\omega$ and $s$.

This expression has been used by some other authors [8]. Otherwise, to take into account both skin and proximity effects some authors have shown how to determine from simulation [9] or from measurement [10] copper losses by using a series resistor. Harburg [11] have modeled $\mathrm{AC}$ losses and compared them with $3 \mathrm{D}$ simulations. A new approach to calculate copper losses by using a 2D distribution of the magnetic field was also developed [12]. These various methods underconsider or overestimate strongly the losses and they cannot be applied to every component working at high frequency.

So, a method has been developed in order to determine the losses in integrated components. This method has been succinctly presented in $[13,14]$. In this article, the developed method is presented in detail and compared with several integrated coreless inductors where the serial resistance of integrated components is determined from measurement or from simulation.

The resistance extraction at low frequencies is obtained very exactly. However, beyond some tens of $\mathrm{MHz}$, it is impossible to measure exactly the resistance using an impedance analyzer. A solution consists in substituting the two-port current-voltage approach by an approach based upon scattering parameters. Thus, only scattering parameters can be obtained by using a Vector Network Analyzer (VNA). Software solutions based on finite element such as High Frequency Structure Simulator (HFSS) also calculate scattering parameters. Well known equations are available to convert $S_{i j}$ parameters into $Y_{i j}$ or $Z_{i j}$ parameters [15].

In this article, coreless inductors (Fig. 1) are chosen in order to have only the resistance parameter which depends on the frequency and allow the developed method to be validated. Afterward, this method can be used for integrated inductors with magnetic material.

Several authors [16-21] modelled the planar inductors by an RLC electrical circuit. Figure 2 shows a coreless inductor model: $L$ is the inductance, $C_{1}$ and $C_{2}$ represent the capacitive coupling between the spiral and the ground plane and $C_{12}$ takes into account the capacitive coupling between turns. The only resistance $\mathbf{r}_{(\mathbf{f})}$ is depending on

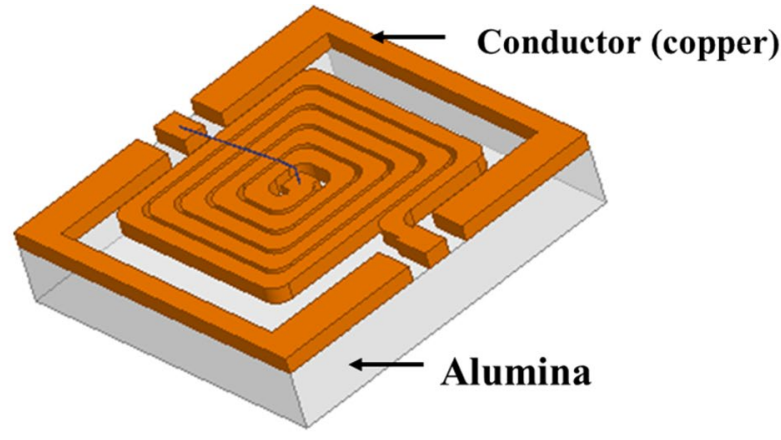

(a)

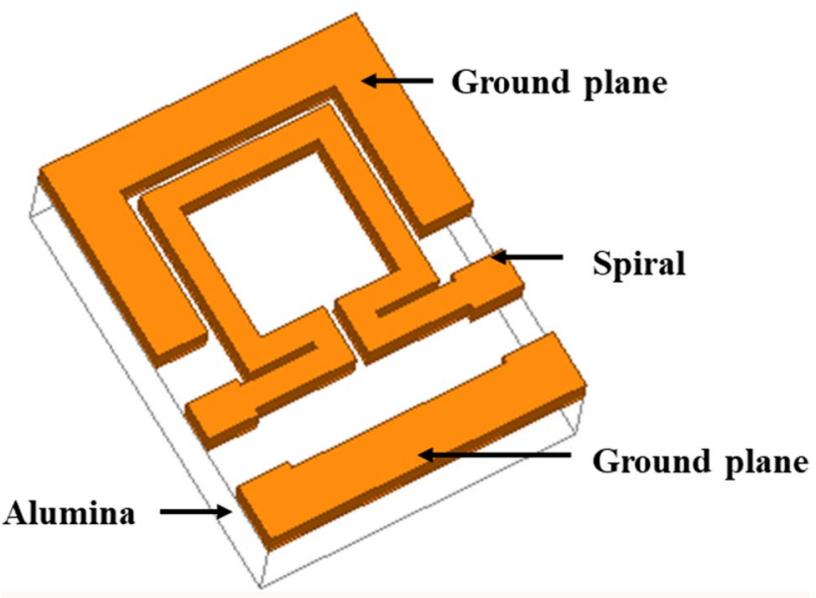

(b)

Fig. 1 Coreless inductors, a spiral inductor with 5 turns b spiral inductor with 1 turn

the frequency in order to model both skin and proximity effects.

\section{Determination of the model parameters}

\subsection{Equivalent electrical circuit}

Inductors are considered as a two-port network (Fig. 3). They are usually expressed in matrix notation which establishes relations between the current-voltage variables:

$V_{1}$ voltage across port $1, I_{1}$ current into port $1, V_{2}$ voltage across port 2 and $\mathrm{I}_{2}$ current into port 2 .

The relationship between the port voltages, port currents and the Y-parameter matrix is given by:

$\left[\begin{array}{l}I_{1} \\ I_{2}\end{array}\right]=\left[\begin{array}{l}Y_{11} Y_{12} \\ Y_{21} Y_{22}\end{array}\right]\left[\begin{array}{l}V_{1} \\ V_{2}\end{array}\right] \quad\left\{\begin{array}{l}I_{1}=Y_{11} \cdot V_{1}+Y_{12} \cdot V_{2} \\ I_{2}=Y_{21} \cdot V_{1}+Y_{22} \cdot V_{2}\end{array}\right.$

Four constant elements $\left(L, C_{1}, C_{2}\right.$ and $\left.C_{12}\right)$ and one frequency dependent, $r$, parameters have to be determined. 


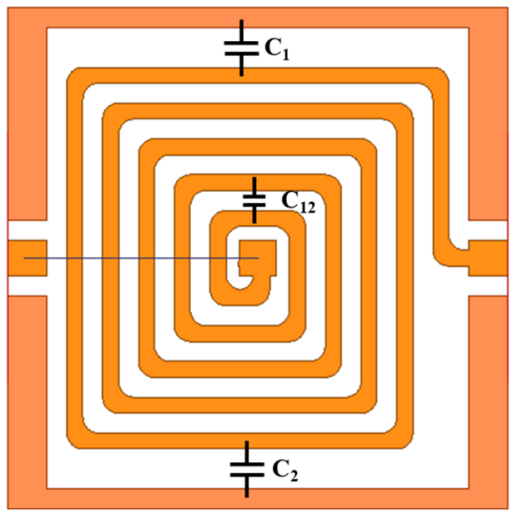

(a)
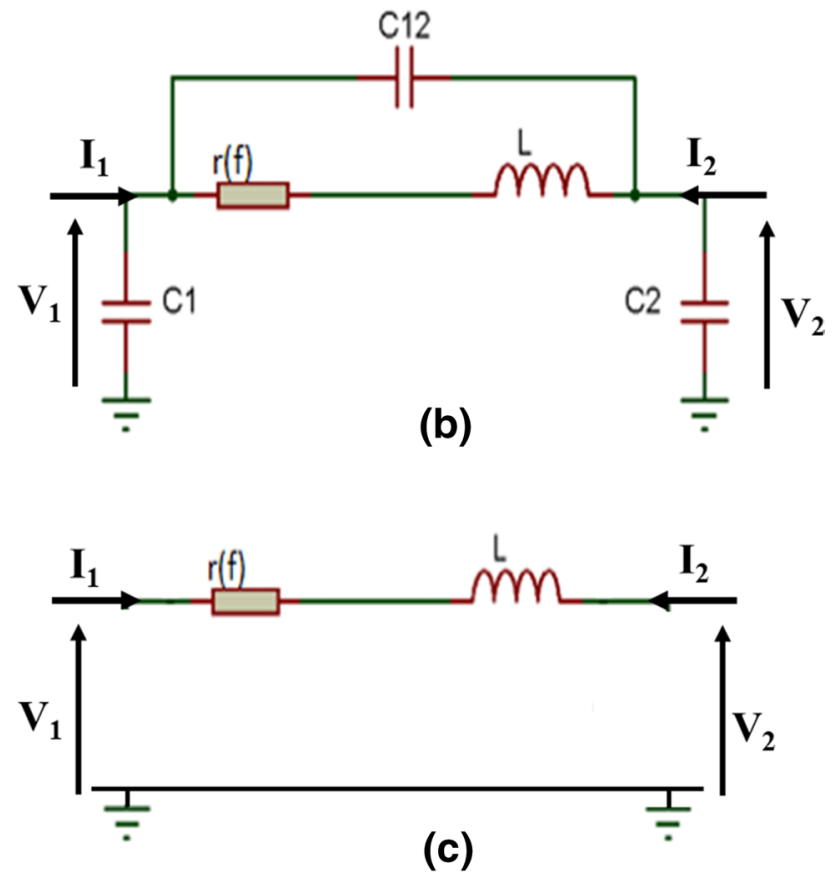

Fig. 2 Coreless inductor model a capacitance representation on the spiral inductor, $\mathbf{b}$ electrical model at high frequencies, c electrical model at low frequencies

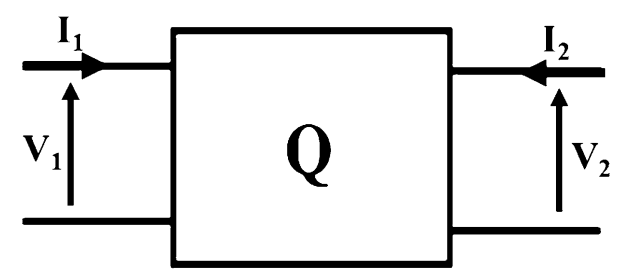

Fig. 3 Two-port network (quadrupole)

$L$ is constant because no magnetic material is used for this inductor and the loss tangent of the substrate is negligible [22]. Capacitances are also constant because the substrate permittivity is constant on the entire frequency range.

Using impedance matrix of the full inductor, the model parameters $L, C_{1}, C_{2}$ and $C_{12}$ can be determined.

$Y_{11}=\frac{1}{r_{(f)}+j L 2 \pi f}+j\left(C_{1}+C_{12}\right) 2 \pi f$

$Y_{12}=Y_{21}=-\left(\frac{1}{r_{(f)}+j L 2 \pi f}+j C_{12} 2 \pi f\right)$

$Y_{22}=\frac{1}{r_{(f)}+j L 2 \pi f}+j\left(C_{2}+C_{12}\right) 2 \pi f$

These four constant parameters are determined as shown in Fig. 4. In the linear part of the curve (slope of $-20 \mathrm{~dB} / \mathrm{dec}$ ade) that represents the evolution of the $Y_{12}$-parameter versus frequency, one can state that $Y_{12}$ is purely inductive. In these conditions,

$L \omega=\frac{1}{\left|Y_{12}\right|}$

and

$L=\frac{10^{-Y_{12} \mathrm{~dB} / 20}}{2 \pi f}$

The resonance frequencies of $Y_{12}, Y_{11}$ and $Y_{22}$ admittances allow the capacitances $C_{12}, C_{1}$ and $C_{2}$ respectively to be calculated. The frequency resonance of an $\mathrm{LC}$ network is related to $L$ and $C$ values:

$f_{0}=1 / 2 \pi \sqrt{L C}$

In these conditions, capacitances are expressed below:

$C_{12}=\frac{1}{f_{0 Y_{12}}^{2} 4 \pi^{2} L}$

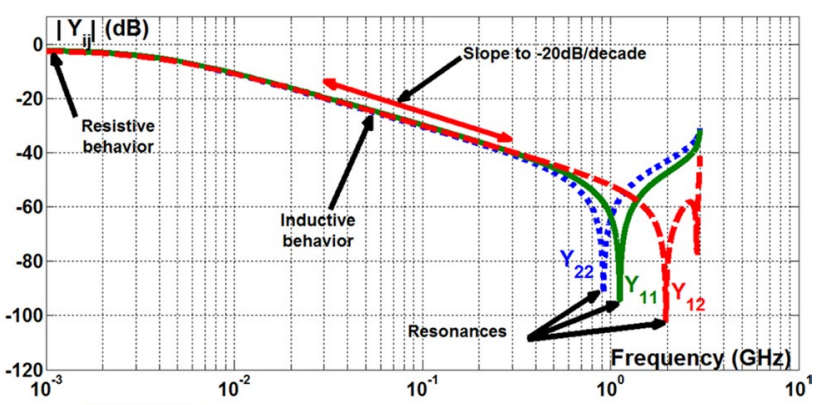

Fig. 4 Simulated $\mathrm{Y}_{\mathrm{ij}}$ admittance curves 
$C_{12}+C_{1}=\frac{1}{f_{0 Y_{11}}^{2} 4 \pi^{2} L}$

$C_{12}+C_{2}=\frac{1}{f_{0 Y_{22}}^{2} 4 \pi^{2} L}$

$f_{O Y 12}, f_{O Y 11}, f_{0 Y 22}$ are parallel resonance frequencies of $Y_{12}$, $\mathrm{Y}_{11}$ and $\mathrm{Y}_{22}$ parameters.

\subsection{Accuracy and frequency range}

A careful consideration should therefore be given to the choice of the frequency domain in order to determine the $r_{(f)}$ value. Calculation of $Y_{i j}$ parameters using scattering parameters can lead to significant errors. In order to illustrate this problem, $Y_{12}$ parameter has been calculated with inaccurate $S_{i j}$ parameters. Figure 5 shows $Y_{12}$ parameter that was calculated with 3 sets of different $S_{i j}$ parameters: $S_{i j}$ (the correct value), $0.99 S_{i j}$ (with an error of $1 \%$ ) and $0.95 \mathrm{~S}_{\mathrm{ij}}$ (with an error of $5 \%$ ).

Important errors can be observed at low frequencies that means $Y_{12}$ parameter has not to be used in this frequency range.

So, the resistance can be measured with an LRC-meter or calculated.

The resistance $\mathbf{r}_{(\mathbf{f})}$ is frequency dependent and has to be determined in 3 frequency domains:

1. at very low frequencies (or DC) without using $Y_{12}$

2. at medium frequencies (capacitive couplings are negligible) and the impedances $r$ and $L \omega$ are in the same order of magnitude (before the slope of $-20 \mathrm{~dB} / \mathrm{dec}$ ade)

3. at resonant frequencies i.e. high frequencies.

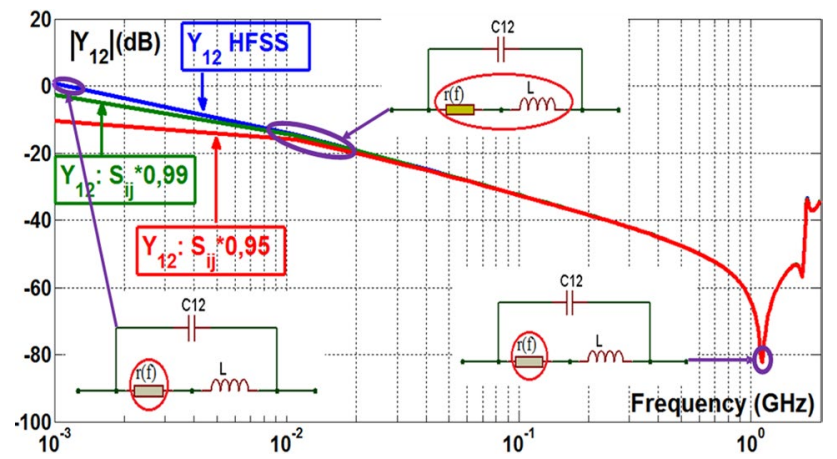

Fig. $5 Y_{12}$ versus frequency: accuracy and frequency range

\subsubsection{At very low frequencies (or DC)}

The very low frequencies resistance can be either calculated or measured. If the 3D finite element analysis software is not able to give an accurate value at low frequencies, the DC resistance can be calculated using [10]:

$R_{D C}=\frac{\rho l}{S}$

where $\rho$ is the copper resistivity, I the length of the wire and $\mathrm{S}$ the copper section.

LCR-meter or Impedance-meter are suitable equipment to measure a low frequency resistance by using a 4-wires method.

\subsection{At low-medium frequencies}

Figure 6 shows the equivalent circuit for $Y_{12}$ parameter in this frequency range. At low-medium frequencies capacitive couplings $\left(C_{1}, C_{2}\right.$ and $\left.C_{12}\right)$ are negligible and only consider the two elements $r_{(f)}$ and $L$ can be considered.

So, the expression of the $Y_{12}$ parameter is:

$Y_{12}=-\frac{1}{r_{(f)}+j L \omega}$

From Eq. (14), the real part of this expression is equal to:

$\operatorname{Real}\left(Y_{12}\right)=-\frac{r_{(f)}}{r_{(f)}^{2}+L^{2}(2 \pi f)^{2}}$

In order to simplify the previous expression, write out the following equation:

$\alpha=\operatorname{Real}\left(Y_{12}\right)$

Then, the equation becomes:

$\alpha r^{2}+r+\alpha L^{2} \omega^{2}=0$

The discriminant of this equation always being positive, two real solutions exist:

$r_{1}=\frac{-1-\sqrt{1-4 \alpha^{2} L^{2} \omega^{2}}}{2 \alpha}$

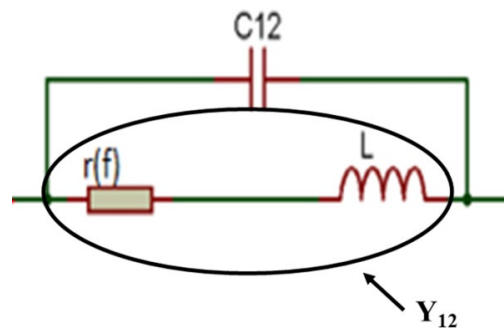

Fig. $6 Y_{12}$ equivalent circuit model at low-medium frequencies 
$r_{2}=\frac{-1+\sqrt{1-4 \alpha^{2} L^{2} \omega^{2}}}{2 \alpha}$

In order to determine the right solution, it is necessary to express the two solutions depending on the frequency.

$\Rightarrow$ At low frequencies $4 \alpha^{2} L^{2} \omega^{2} \rightarrow 0$ that implies:

$r_{1}=\frac{-1-\sqrt{1}}{2 \alpha}$

$r_{1}=-1 / \alpha$ is the right solution and $r_{2}=\frac{-1+\sqrt{1}}{2 \alpha}=0$ is not the solution.

$\Rightarrow$ At low-medium frequencies $L \omega \gg r$, then

$\alpha=\frac{-r}{L^{2} \omega^{2}}$

that implies:

$r_{2}=\frac{-1+\sqrt{1-4\left(\frac{-r}{L^{2} \omega^{2}}\right)^{2} L^{2} \omega^{2}}}{2 \alpha}=\frac{-1+\sqrt{1-4 \frac{r^{2}}{L^{2} \omega^{2}}}}{2 \alpha}$

The limited development of $\sqrt{1-\varepsilon}$ is $1-\varepsilon / 2$, then

$\boldsymbol{r}_{2}=\frac{-1+\left[1-4 \frac{\boldsymbol{r}^{2}}{L^{2} \omega^{2}} * \frac{1}{2}\right]}{2 \boldsymbol{\alpha}} \Rightarrow \boldsymbol{r}_{2}=\frac{-2 \frac{\boldsymbol{r}^{2}}{L^{2} \omega^{2}}}{2 \frac{-\boldsymbol{r}}{L^{2} \omega^{2}}}$

$r_{2}=r$ is the right solution and $r_{1}$ is not the right solution.

Thus, for low-medium frequencies, the physical solution of this quadratic equation is given by

$r=\frac{-1+\sqrt{1-4 \alpha^{2} L^{2}(2 \pi f)^{2}}}{2 \alpha}$

\subsubsection{At resonant frequencies i.e. high frequencies}

At high frequencies no parameter can be neglected. In these conditions, it is very difficult to extract the $r$ value except at the resonant frequency of each Y-parameter. Indeed at the resonant frequency the magnitude of the $Y$-parameter curve only depends on $r$ value. By using a curve fitting procedure, the $r$ value at the resonant frequency can be determined.

The presented method which consists in determining the $\mathbf{r}_{\text {(f) }}$ serial resistance in 3 different frequency ranges, has been applied to different coreless inductors.

\section{Devices and manufacturing, simulation and measurement}

\subsection{Devices under test}

Many inductors were manufactured by changing different parameters such as number of turns ( $\mathbf{N}$ from 1 to 15 ), ribbon width (W from 100 to $400 \mu \mathrm{m}$ ) and space width (S from 50 to $400 \mu \mathrm{m}$ ). Figure 7 illustrates the main dimensions of coreless inductors.

For instance different space widths between turns allow the influence of the distance between turns on proximity effects to be studied. All the devices were manufactured on alumina substrate $\left(\mathrm{Al}_{2} \mathrm{O}_{3}\right)$. At studied frequencies (below $\mathrm{GHz}$ ) this material exhibits no losses ( $\operatorname{tg} \delta<10^{-3}$ [22]). It can be considered as perfect. In these conditions only the relative permittivity $\varepsilon_{\mathrm{r}}$ of the substrate is considered for simulation. Figure 8 shows a picture of some fabricated devices. Different square spirals with a ground plane were manufactured in order to use Ground Signal Ground (GSG) probe for the device characterization.

\subsection{Inductor manufacturing}

The process concerns copper deposition, photolithography and wet etching to obtain the final pattern of the square spiral inductor.

First of all, a copper layer is deposited with a thickness chosen according to the desired device (usually

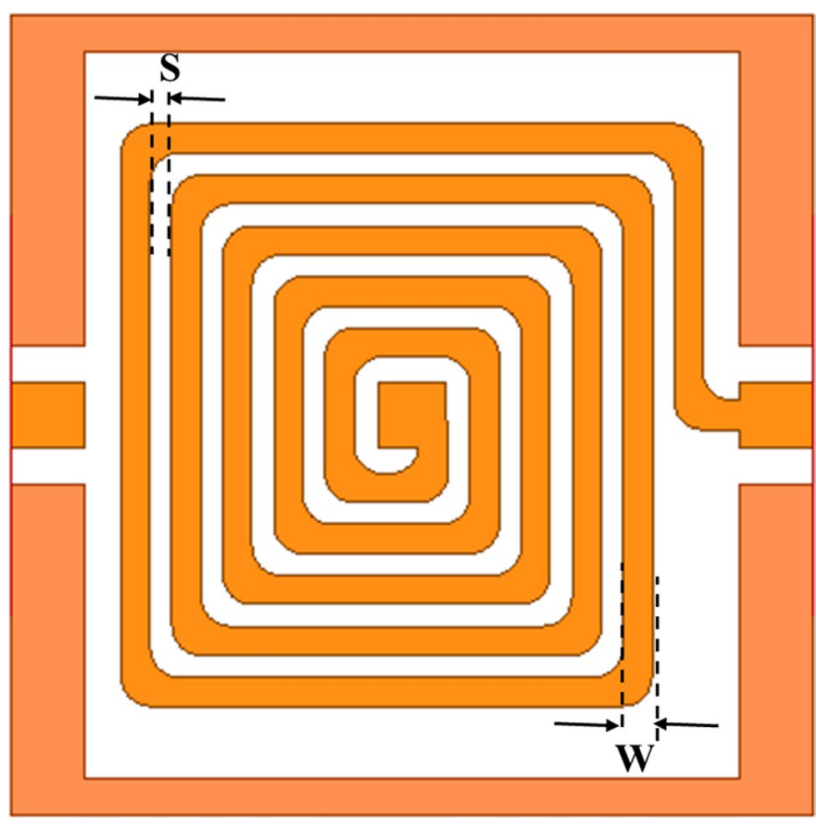

Fig. 7 Main dimensions of inductors 


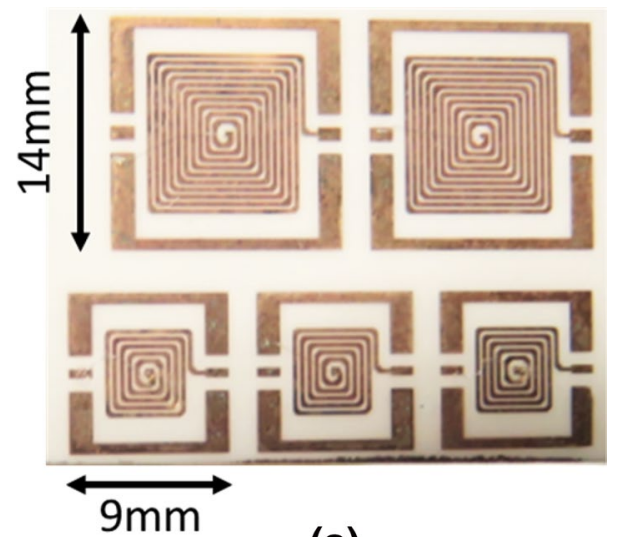

(a)

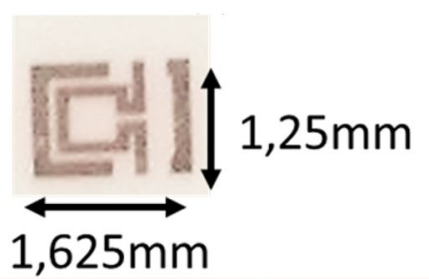

(b)

Fig. 8 Coreless inductors on alumina substrate $\mathbf{a}$ inductors with 5 and 10 turns, $\mathbf{b}$ inductor with 1 turn

between 1 and $20 \mu \mathrm{m}$ ). The conductive material (copper) deposited by RF sputtering is chosen for its high electrical conductivity.

The spiral pattern manufacturing is obtained in a clean room by conventional photolithography techniques that can be summarized succinctly as follow:

- Deposition of a photoresist layer by spin coating (SPR 505).

- Annealing at $110^{\circ} \mathrm{C}$ using a hot plate for $90 \mathrm{~s}$.

- Insulation during $12 \mathrm{~s}$.

- Second annealing at $110^{\circ} \mathrm{C}$ using an oven for $90 \mathrm{~s}$.

- Developing and removing photoresist layer

- Final annealing at $110^{\circ} \mathrm{C}$ using an oven for $4 \mathrm{~min}$.

These steps are followed by wet etching in a solution of ferric chloride at $30^{\circ} \mathrm{C}$. The etching time depends on the concentration of ferric chloride, the temperature of the solution and the thickness of the deposited layer. The etching is generally performed in less than $1 \mathrm{~min}$. In order to prevent any oxidation of the copper ribbons a thin gold layer is electroplated on the copper ribbons.

The last step concerns the connection between the inner central pad and the outer one. This connection is performed by using a wire bonding.

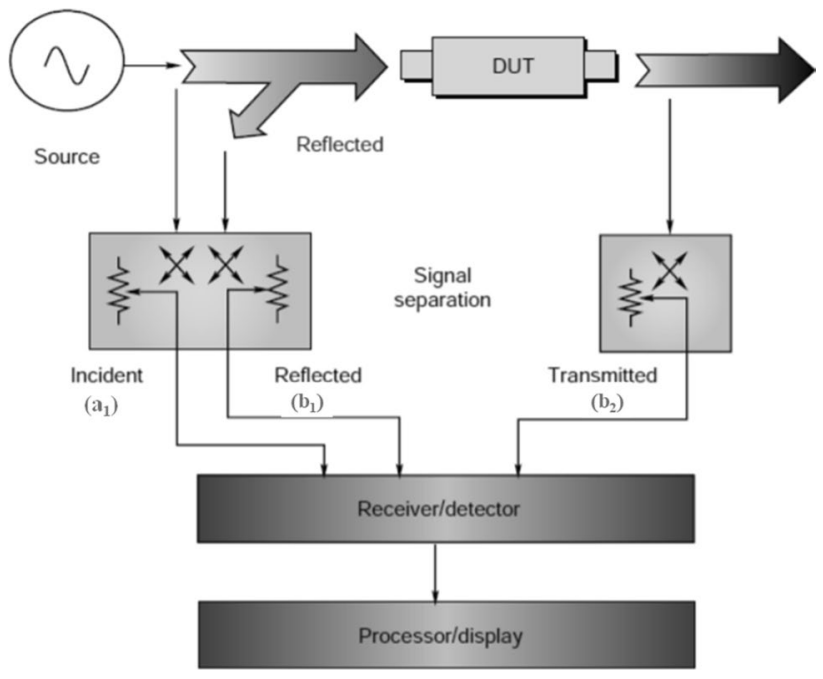

Fig. 9 Operating principle diagram of the vector network analyser

\subsection{Simulation}

Simulations are carried out with HFSS, a 3-D full-wave, frequency domain electromagnetic field solver based on the finite element method (FEM). In order to take into account both skin and proximity effects the copper conductors are also meshed in volume. Moreover the device under test is positioned in an important air box. Boundary conditions are defined as a perfect conductor (Perfect E).

\subsection{Measurement set-up}

Measurements are performed using a vector network analyzer (Rohde\&Schartz ZVA67) and a probing system. This equipment allow the devices to be characterized on a broad band frequency ( $10 \mathrm{MHz}$ to $2 \mathrm{GHz}$ ). The operating principle of the vector network analyzer consists in emitting a sinusoidal signal with constant amplitude and variable frequency on the device to determine the dispersion parameters $S$ of this device (Fig. 9).

This diagram explains the principle for measuring $S_{11}$ and $S_{21}$ parameters. In order to obtain the complete dispersion matrix, the source is activated in the second port. By switching the source on both ports, the reflection and transmission parameters $\mathrm{S}_{11}, \mathrm{~S}_{21}, \mathrm{~S}_{12}$ and $\mathrm{S}_{22}$ can be measured.

In order to minimize measurement errors, $\mathrm{S}_{\mathrm{ij}}$ parameters are measured with a prior OSTL (Open Short Thru Load) calibration. This calibration allows an impedance reference at the end of the probe to be equal to $50 \Omega$.

The measurement results are expressed as $S_{\mathrm{ij}}$ parameters. To analyze these results, intermediate relations are used to obtain $Y_{\mathrm{ij}}$ parameters [15]. 
Fig. 10 Measured series resistance $r_{(f)}$ versus frequency

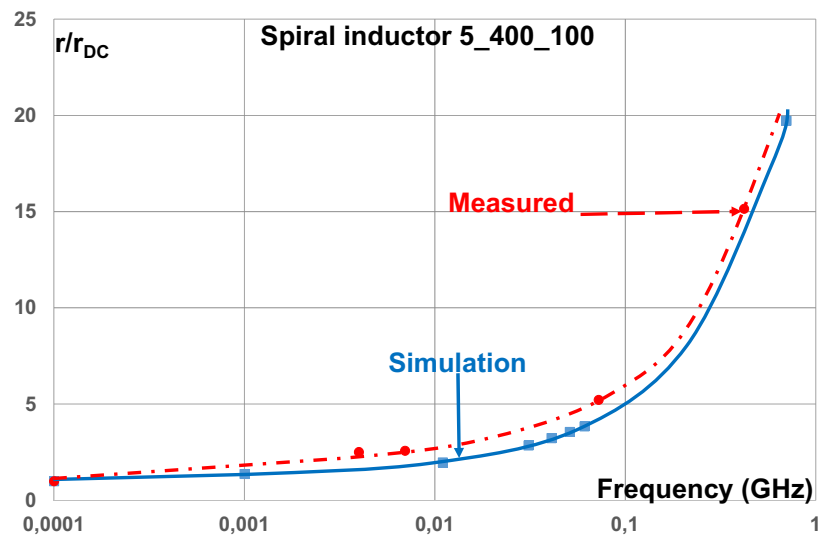

Fig. 11 Comparison between extracted resistances from simulation and measurement for inductor 5 turns $400 \mu \mathrm{m}$ ribbon width_100 $\mu \mathrm{m}$ turn spacing

\section{Results}

In order to illustrate our approach, Fig. 10 shows an example of the variation of the resistance $r_{(f)}$ versus frequency. This curve is drawn for a coreless inductor which exhibits the following characteristics: - number of turns (N): 5-square spiral-copper thickness (e): $5 \mu \mathrm{m}$-ribbon width (W): $400 \mu \mathrm{m}$-space width (S): $50 \mu \mathrm{m}$. This device is noted 5-400-50. The two high-frequency points marked by a triangle were obtained from the resonances of the parameters $Y_{11}$ and $Y_{22}$.

\subsection{Comparison between simulation and measurement}

Comparisons between extracted resistances from both simulation and measurement are shown in Figs. 11, 12,13 and 14 for four inductors which exhibit 1, 5, 10 and 15 turns. The dashed curves show the ratio $r_{(f) /} r_{D C}$
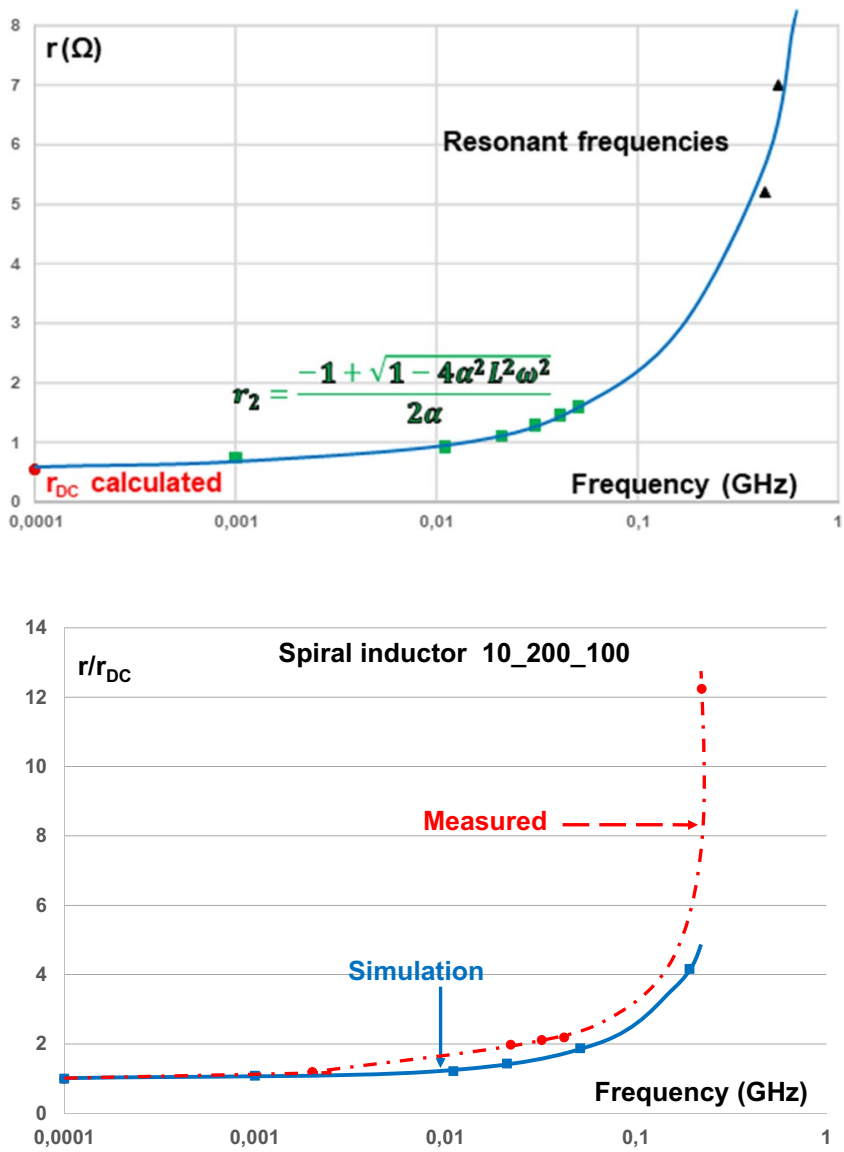

Fig. 12 Comparison between extracted resistances from simulation and measurement for inductor 10 turns_200 $\mu \mathrm{m}$ ribbon width $100 \mu \mathrm{m}$ turn spacing

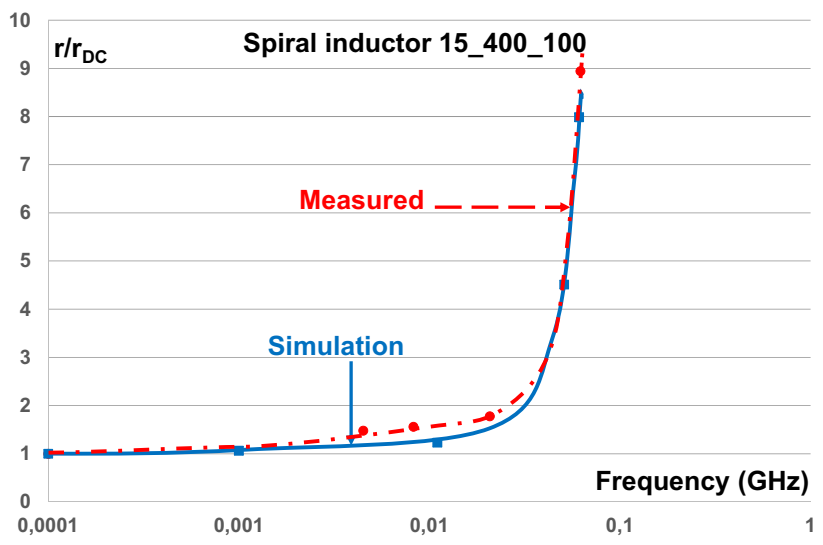

Fig. 13 Comparison between extracted resistances from simulation and measurement for inductor 15 turns_400 $\mu \mathrm{m}$ ribbon width 100 $\mu \mathrm{m}$ turn spacing 


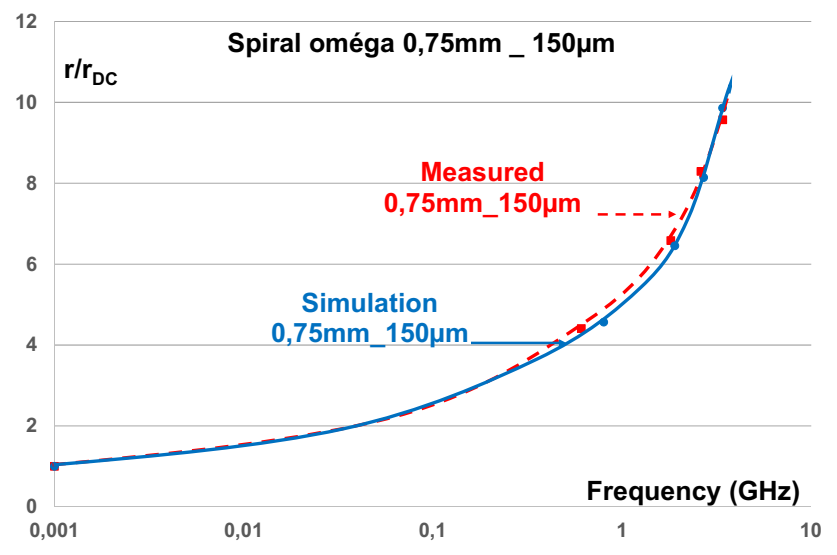

Fig. 14 Comparison between extracted resistances from simulation and measurement for inductor 1 turn_0.75 mm length _ $150 \mu \mathrm{m}$ ribbon width

determined from measurements while the solid curves show $\mathbf{r}_{(f) /} \mathbf{r}_{\text {DC }}$ determined from simulation (HFSS).

A good agreement between simulated and measured curves is observed for each inductor. Thus, these results allow the developed method to be validated. On the other hand, these results show that either simulated curves or measured can be used for determine the evolution of the resistance depending on the frequency.

\subsection{Method for separating skin effects and proximity effects}

In order to illustrate skin effects and proximity effects, Fig. 15 compares two normalized resistance $r / r_{D C}$ as a function of frequency. These two curves correspond to the same device (all geometric parameters are identical).
The curve $\left(C_{1}\right)$ is obtained by using our approach in order to take into account all winding high-frequency effects.

The curve $\left(C_{2}\right)$ shows the increase of the ratio $r_{(f) / r} r_{D C}$ which is only due to skin effects (proximity effects are not taken into account). The expression given by Yue [6] (1) is used to calculate $r_{(f)}$ :

From Fig. 15 curve $C_{1}$ exhibits a higher increase than curve $C_{2}$ which only considers skin effects. This confirms that both skin and proximity effects are taken into account in our novel method.

In order to validate the previous assumption, Fig. 16 shows the ratio $r_{(f) /} r_{D C}$ versus frequency for different turn spacing, the other parameters are kept identical:

- Curve $\left(C_{1}\right)$ the turn spacing is equal to $50 \mu \mathrm{m}$,

- Curve $\left(C_{2}\right)$ the turn spacing is equal to $100 \mu \mathrm{m}$,

- Curve $\left(C_{3}\right)$ the turn spacing is equal to $400 \mu \mathrm{m}$

Figure 16 shows that $\mathrm{r}_{(\mathrm{f}) /}, r_{\mathrm{DC}}$ ratios depend on turn spacing. The smaller the space between turns is the higher the ratio $r_{(f) /} r_{D C}$ is. That demonstrates proximity effects are taken into account.

In these conditions, skin and proximity effects can be separated as illustrated on Fig. 17.

- The curve $\left(C_{1}\right)$ shows the resistance $r_{D C}$ that can be either measured by using a low frequency equipment such as LCR meter or calculated $\left(r_{D C}=\rho \frac{l}{S}\right)$

- The curve $\left(C_{2}\right)$ that only takes into account skin effect is obtained from Yue's expression (1).

- The curve $\left(C_{3}\right)$ taking into account both skin and proximity effects is obtained either from measurement or from simulation.

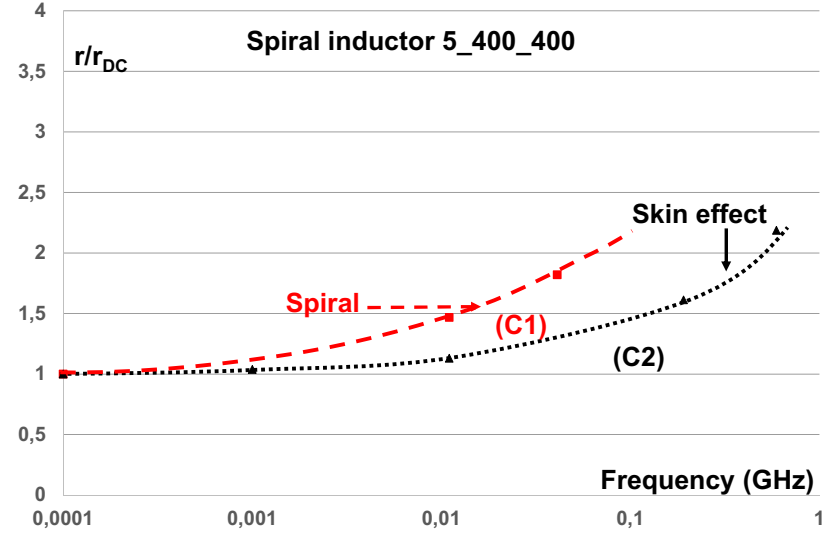

Fig. $15 r_{(f) / r_{D C}}$ versus frequency taking into account skin effect (C2) and skin and proximity effects (C1) for inductor 5 turns_ $400 \mu \mathrm{m}$ ribbon width $400 \mu \mathrm{m}$ turn spacing

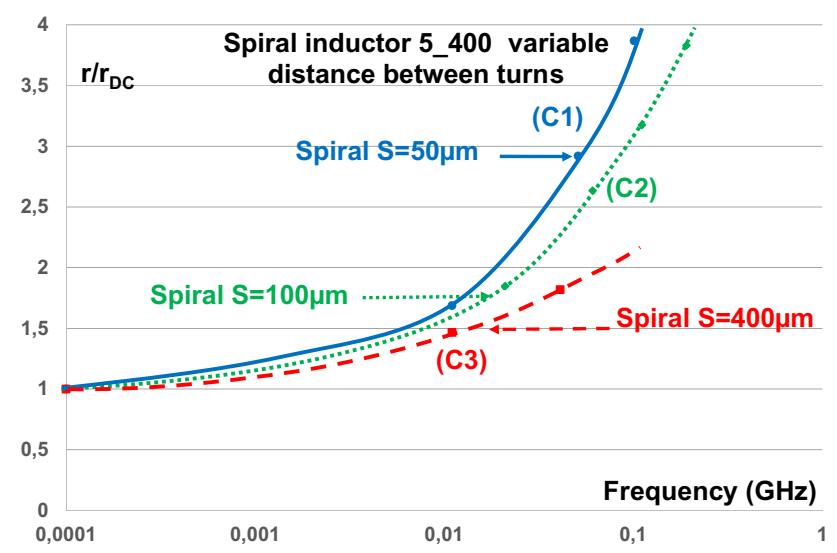

Fig. $16 r_{(f) /} r_{D C}$ versus frequency taking into account skin and proximity effects for 3 inductors 5 turns_ $400 \mu \mathrm{m}$ ribbon width and different turn spacing $(50 \mu \mathrm{m}, 100 \mu \mathrm{m}$ and $400 \mu \mathrm{m})$ 
Fig. 17 Loss separation for inductor 5 turns_ $400 \mu \mathrm{m}$ ribbon width $400 \mu \mathrm{m}$ turn spacing

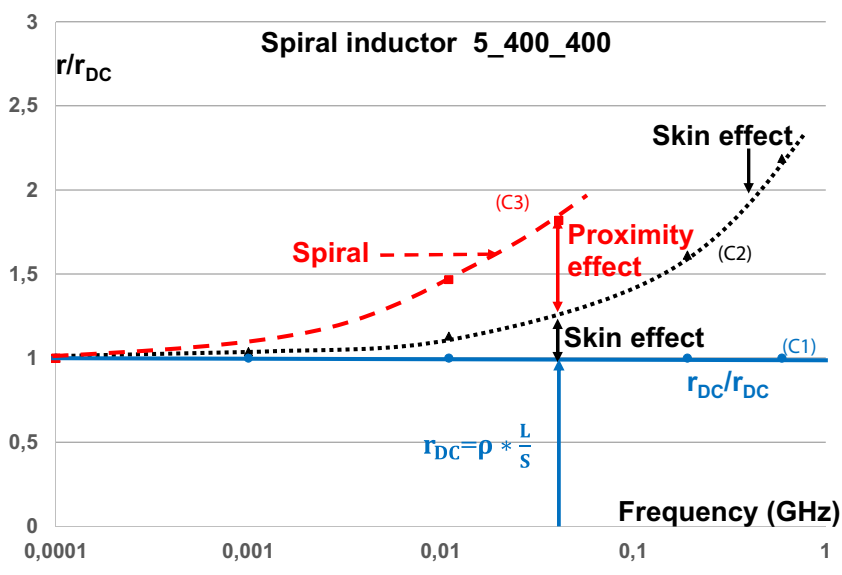

7. Kuhn WB, Ibrahim NM (2000) Analysis of current crowding effects in multiturn spiral inductors. IEEE Trans Microw Theory Tech 49(1):31-38

In this article, winding loss determination for integrated inductors has been demonstrated. The presented approach is different from those encountered in literature and allows the series resistance from measured or simulated scattering parameters $S_{i j}$ to be determined. Winding losses are taken into account by means of a series resistance which depends on frequency. Simulation and measurements have been carried out and a good agreement is observed. This method allows losses to be separated in two parts: skin effect and proximity effect for coreless inductors.

\section{Compliance with ethical standards}

Conflict of interest The authors declare that they have no conflict to interest.

\section{References}

1. Dowell PL (1966) Effects of eddy currents in transformer windings. Proc Inst Electr Engin 113(8):1387-1394

2. Ferreira JA (1994) Improved analytical modeling of conductive losses in magnetic components. IEEE Trans Power Electron 9(1):127-131

3. Xi N, Sullivan CR (2003) An improved calculation of proximityeffect loss in high-frequency windings of round conductors. In: Procedings of the IEEE 34th annual conference on power electronics specialist

4. Roßkopf A, Bar E, Joffe C (2014) Influence of inner skin- and proximity effects on conduction in litz wires. IEEE Trans Power Electron 29(10):5454-5461

5. Chen W, Yan Y, Hu Y, Lu Q (2003) Model and design of PCB parallel winding for planar transformer. IEEE Trans Magn 39(5):3202-3204

6. Yue CP, Wong SS (2000) Physical modeling of spiral inductors on silicon. IEEE Trans Electron Devices 47(3):560-568
8. Lee KK, Bryant C, Törmänen M, Sjöland H (2009) A 65-nm CMOS ultra-low-power LC quadrature VCO. In: Proceedings of the NORCHIP conference

9. Kaluza M, Napieralski A (2008) Frequency sampling approach to the problem of silicon integrated spiral inductors modeling. Bull Pol Acad Sci 56(1):21-27

10. Kuhn WB, Boutz AP (2010) Measuring and reporting high quality factors of inductors using vector network analyzers. IEEE Trans Microw Theory Tech 58(4):1046-1055

11. Harburg DV, Qiu J, Sullivan CR (2012) An improved AC loss model for the optimization of planar-coil inductors. In: IEEE 13th workshop on control and modeling for power electronics

12. Wang N, O'Donnell T, O'Mathuna C (2013) An improved calculation of copper losses in integrated power inductors on silicon. IEEE Trans Power Electron 28(8):3641-3647

13. Abderahim A, Mahamat AT, Chatelon JP, Pietroy D, Capraro S, Rousseau JJ (2016) Approach of copper losses determination in planar windings. Electron Lett 52(12):1050-1052

14. Abderahim A,Mahamat AT, Capraro S, Moungache $S$, Chatelon JP, Pietroy D, Rousseau JJ (2016) Determination of copper losses from measurement and simulation in planar windings. In: Proceedings of the design, test, integration and packaging of MEMS/MOEMS

15. Frickey DA (1994) Conversions between $S, Z, Y, H, A B C D$, and $T$ parameters which are valid for complex source and load impedances. IEEE Trans Microw Theory Tech 42(2):205-211

16. Ooi B-L, Dao-Xian X, Kooi P-S, Lin F-J (2002) An improved prediction of series resistance in spiral inductor modeling 50(9):2202-2206

17. Chen J, Liou JJ (2004) On-chip spiral inductors for RF applications: an overview. J Semicond Technol Sci 4(3):149-167

18. Talwalkar NA, Yue CP, Wong SS (2005) Analysis and synthesis of on-chip spiral inductors. IEEE Trans Electron Devices 52(2):176-182

19. Han Y, Cheung G, Li A, Sullivan CR, Perreault DJ (2012) Evaluation of magnetic materials for very high frequency power applications. IEEE Trans Power Electron 27(1):425-435

20. Cao Y, Groves RA, Zamdmer ND, Plouchart JO, Wachnik RA, Huang X, King TJ, Hu C (2020) Frequency-independent equivalent-circuit model for on-chip spiral inductors. In: Proceedings of the IEEE custom integrated circuits conference with eddy current effect. IEEE Trans Microw Theory Tech 
21. Mu M, Li Q, Gilham DJ, Lee FC, Ngo KDT (2014) New core loss measurement method for high-frequency magnetic materials. IEEE Trans Power Electron 29(8):4374-4381

22. Rajab KZ, Naftaly M, Linfield EH, Nino JC, Arenas D, Tanner D, Mittra R, Lanagan M (2008) Broadband dielectric characterization of aluminum oxide $\left(\mathrm{Al}_{2} \mathrm{O}_{3}\right)$. J Micro Electr Pack 5:101-106
Publisher's Note Springer Nature remains neutral with regard to jurisdictional claims in published maps and institutional affiliations. 